\title{
Information-Theoretic Study of Voting Systems
}

\author{
Eitan Yaakobi*, Michael Langberg ${ }^{\dagger}$, and Jehoshua Bruck* \\ *Electrical Engineering Department, California Institute of Technology, Pasadena, CA 91125, U.S.A \\ ${ }^{\dagger}$ Department of Mathematics and Computer Science, Open University of Israel, Raanana 43107, Israel \\ \{yaakobi,bruck\}@caltech.edu,mikel@openu.ac.il
}

\begin{abstract}
The typical paradigm in voting theory involves $n$ voters and $m$ candidates. Every voter ranks the candidates resulting in a permutation of the $m$ candidates. A key problem is to derive the aggregate result of the voting. A popular method for vote aggregation is based on the Condorcet criterion. The Condorcet winner is the candidate who wins every other candidate by pairwise majority. However, the main disadvantage of this approach, known as the Condorcet paradox, is that such a winner does not necessarily exist since this criterion does not admit transitivity. This paradox is mathematically likely (if voters assign rankings uniformly at random, then with probability approaching one with the number of candidates, there will not be a Condorcet winner), however, in real life scenarios such as elections, it is not likely to encounter the Condorcet paradox. In this paper we attempt to improve our intuition regarding the gap between the mathematics and reality of voting systems. We study a special case where there is global intransitivity between all candidates. We introduce tools from information theory and derive an entropy-based characterization of global intransitivity. In addition, we tighten this characterization by assuming that votes tend to be similar; in particular they can be modeled as permutations that are confined to a sphere defined by the Kendalls $\tau$ distance.
\end{abstract}

\section{INTRODUCTION}

The problem of rank aggregation appears in many contexts, such as social choice decisions, web search algorithms, and voting theory. In the general model, there are $n$ voters and $m$ candidates. For example, in the context of web search algorithms, each voter can be associated with a ranking of relevant web pages (i.e., the candidates). There are several models for the voters to assign their rankings. For example, they can vote to a single candidate, top $k$ candidates, top and bottom candidates etc. Given the $n$ rankings, a central and well studied goal is to construct a final ranking which best represents the rankings of the voters. In particular, such a final ranking will identify a winner aggregate candidate. There are several voting rules to construct the final ranking such as the plurality, anti-plurality, Borda, Copeland, Maximin, Dodgson, and more. For an excellent historic overview on social choice we refer the reader to [3] and for a nice survey [5].

In this work we solely consider the model where the ranking of every voter is a full order of the candidates, i.e. a permutation $\sigma$ in $\mathbb{S}_{m}$, the set of all $m$ ! permutations. The vector of all rankings is denoted by $\Sigma=\left(\sigma_{1}, \ldots, \sigma_{n}\right) \in\left(\mathbb{S}_{m}\right)^{n}$ and is called a profile. The $i$-th candidate is winning the $j$-th candidate if it is ranked higher by more voters according to pairwise comparison (for simplicity assume that ties cannot appear). A natural approach to choose a winner, proposed in 1785 by Marquis de Condorcet and is called the Condorcet criterion, is to pick the candidate who is winning every other candidate according to this pairwise majority [1]; such a candidate is called the Condorcet winner. However, a Condorcet winner does not necessarily exist; this is known as the Condorcet paradox. The paradox states the counter intuitive phenomena that well defined input rankings may result in the lack of an aggregate winner, under certain natural definitions in which such a winner would be expected. More specifically, if the $i$-th candidate is winning the $j$-th candidate and the $j$-th candidate is winning the $k$-th candidate then the $i$-th candidate is not necessarily winning the $k$-th candidate. That is, the Condorcet criterion does not preserve transitivity. This intransitivity may introduce a cycle of length three and similarly longer cycles are defined. One extension of the Condorcet winner is known as the Smith set [9]. It is said that $S$ is a winning set if every candidate in $S$ is winning every candidate not in $S$, and the smallest set $S$ which satisfies this condition is called the Smith set. As opposed to a Condorcet winner, the Smith set always exist and is unique. A Smith set of size 1 is a Condorcet winner.

It is known that if the number of voters and candidates is large and every voter assigns its permutation uniformly at random from $\mathbb{S}_{m}$ (also known as the impartial culture distribution), then the probability to have a Condorcet winner (and encounter transitivity between the candidates) approaches zero; see e.g. [7], [8]. However, in real life social choice scenarios such as elections it is reported and studied that intransitivity is not likely to happen [10]. For further reading about the Condorcet paradox and its theoretical and practical analysis, we refer the reader to Chapter 1 in [6].

Our goal in this work is to shed more light on the Condorcet paradox. We introduce information-theoretic tools based upon the entropy measure and the Kendall's $\tau$ distance metric in order to introduce conditions to encounter intransitivity between the candidates. Since the tools we study in this work measure the interaction between all the candidates, we study a special case of intransitivity. We say that a profile $\Sigma$ has global intransitivity if there is a cycle between all $m$ candidates. As we present, this definition is equivalent to requiring that the Smith set is the set of all $m$ candidates. Namely, it implies global contradiction between all candidates and thus it is not possible to deduce any partition between them for winning candidates. In analogy to social choice and decision making, no conclusion can be taken about the candidates.

The rest of this paper is organized as follows. In Section II we introduce the information-theoretic tools and measures that will help us in the study of global intransitivity in voting systems. In Section III, we study basic properties which are derived from these tools. Section IV studies implications when a global intransitivity is encountered in a voters profile. One of our claims asserts that if a profile has global intransitivity 
then the sum of Kendall's $\tau$ distances between the rankings of all candidates is at least $\frac{m-1}{4} n^{2}$. In Section V, we show that if the rankings of all candidates belong to a ball of radius no greater than $\lceil m / 2\rceil$, according to the Kendall's $\tau$ distance, then there is no global intransitivity. This bound is tight in the sense that there is an example of global intransitivity when the rankings belong to a ball of radius $\lceil m / 2\rceil+1$.

\section{Definitions And Basic Properties}

Let $n$ be the number of voters and $m$ be the number of candidates. Every voter assigns a full ranking of the $m$ candidates, i.e., a permutation $\sigma \in \mathbb{S}_{m}$, where $\mathbb{S}_{m}$ is the set of all $m$ ! permutations of $m$ candidates. A ranking $\sigma \in \mathbb{S}_{m}$ is denoted by $\sigma=(\sigma(1), \sigma(2), \ldots, \sigma(m))$, where for $1 \leqslant i \leqslant m, \sigma(i)$ is the candidate in the $i$-th rank. Similarly, $\sigma^{-1}(i)$ is the rank of the $i$-th candidate. We denote $i>_{\sigma} j$ if $\sigma^{-1}(i)<\sigma^{-1}(j)$. A profile $\Sigma=\left(\sigma_{1}, \ldots, \sigma_{n}\right) \in\left(\mathbb{S}_{m}\right)^{n}$ is a vector of the rankings of each voter. We say that the $i$-th candidate is winning the $j$-th candidate (or the $j$-th candidate is losing to the $i$-th candidate), and denote it by $i \rightarrow_{\Sigma} j$ if

$$
\left|\left\{\ell \mid i>_{\sigma_{\ell}} j\right\}\right|>\left|\left\{\ell \mid j>_{\sigma_{\ell}} i\right\}\right| .
$$

A profile $\Sigma$ has a Condorcet winner if there exists $1 \leqslant i \leqslant$ $m$ such that the $i$-th candidate is winning every other candidate. A Condorcet loser is defined similarly. Note that a Condorcet winner and loser do not necessarily exist. The natural extension of the definition of a Condorcet winner is called a Smith set [9]. We say that $S \subseteq[m]$, where $[m]=\{1, \ldots, m\}$, is a winning set if for every $i \in S$ and $j \in[m] \backslash S, i$ is winning $j$. A winning set of minimum size is called the Smith set. As opposed to a Condorcet winner, a Smith set always exists and is unique. If a profile $\Sigma$ has a Condorcet winner then the Smith set has a single candidate, the Condorcet winner. A profile $\Sigma$ will be called transitive if there are no three different candidates $i, j, k$ such that $i \rightarrow_{\Sigma} j, j \rightarrow_{\Sigma} k$, and $k \rightarrow_{\Sigma} i$. It is called intransitive if it is not transitive. If $\Sigma$ does not have a Condorcet winner then it is necessarily intransitive, however if it has a Condorcet winner it may still be intransitive. A profile $\Sigma$ has a cycle of length $\ell$ if there are $\ell$ different candidates, $j_{1}, j_{2}, \ldots, j_{\ell}$ such that $j_{1} \rightarrow_{\Sigma} j_{2} \rightarrow_{\Sigma} j_{3} \rightarrow_{\Sigma} \cdots \rightarrow_{\Sigma} j_{\ell} \rightarrow_{\Sigma}$ $j_{1}$. We say that a profile $\Sigma$ has global intransitivity if it has a cycle of length $m$.

Given a profile $\Sigma=\left(\sigma_{1}, \ldots, \sigma_{n}\right) \in\left(\mathbb{S}_{m}\right)^{n}$, its probability ranking matrix $P_{\Sigma}=\left(p_{i, j}\right)_{1 \leqslant i, j \leqslant m} \in[0,1]^{m \times m}$ is defined to be a matrix where the probability $p_{i, j}$ for $1 \leqslant i, j \leqslant m$ is the fraction of voters for which the $j$-th candidate is ranked at the $i$-th rank. Note that the sum of every row and every column of the matrix $P_{\Sigma}$ is one and thus it is a doubly stochastic matrix. Based on the probability ranking matrix, the entropy of every candidate is defined as the entropy of the probabilities of all its rankings. The candidate entropy of the $j$-th candidate, is denoted by $H C_{\Sigma}(j)$ and is defined to be

$$
H C_{\Sigma}(j)=\mathcal{H}\left(p_{1, j}, p_{2, j}, \ldots, p_{m, j}\right),
$$

where $\mathcal{H}$ defines the binary entropy function. Similarly, the ranking entropy of the $i$-th ranking is

$$
H R_{\Sigma}(i)=\mathcal{H}\left(p_{i, 1}, p_{i, 2}, \ldots, p_{i, m}\right) .
$$

A first observation from the structure of the probability matrix is that the sum of candidate entropies and ranking entropies is the same, that is,

$$
\sum_{j=1}^{m} H C_{\Sigma}(j)=-\sum_{j=1}^{m} \sum_{i=1}^{m} p_{i, j} \log \left(p_{i, j}\right)=\sum_{i=1}^{m} H R_{\Sigma}(i) .
$$

Thus, we define the profile entropy of $\Sigma$ to be the sum of all ranking entropies or candidate entropies and denote it by $H(\Sigma)$, where $H(\Sigma)=\sum_{i=1}^{m} H C_{\Sigma}(i)=\sum_{j=1}^{m} H R_{\Sigma}(j)$.

Example 1. Assume there are three candidates and six voters with profile $\Sigma_{1}=\left(\sigma_{1}, \sigma_{2}, \sigma_{3}, \sigma_{4}, \sigma_{5}, \sigma_{6}\right) \in\left(\mathbb{S}_{3}\right)^{6}$, where

$$
\begin{array}{lll}
\sigma_{1}=123, & \sigma_{2}=132, & \sigma_{3}=213, \\
\sigma_{4}=312, & \sigma_{5}=123, & \sigma_{6}=231 .
\end{array}
$$

The probability ranking matrix $P_{\Sigma_{1}}$ is given by

$$
P_{\Sigma_{1}}=\left(\begin{array}{ccc}
1 / 2 & 1 / 3 & 1 / 6 \\
1 / 3 & 1 / 3 & 1 / 3 \\
1 / 6 & 1 / 3 & 1 / 2
\end{array}\right)
$$

The candidate and ranking entropies satisfy

$$
\begin{aligned}
& H C_{\Sigma_{1}}(1)=H C_{\Sigma_{1}}(1)=\mathcal{H}(1 / 2,1 / 3,1 / 6)=1.459, \\
& H C_{\Sigma_{1}}(2)=H R_{\Sigma_{1}}(2)=\mathcal{H}(1 / 3,1 / 3,1 / 3)=1.585, \\
& H C_{\Sigma_{1}}(3)=H R_{\Sigma_{1}}(3)=\mathcal{H}(1 / 6,1 / 3,1 / 2)=1.459,
\end{aligned}
$$

so the profile entropy is $H\left(\Sigma_{1}\right)=4.503$. In this example $1 \rightarrow_{\Sigma_{1}} 2,1 \rightarrow_{\Sigma_{1}} 3,2 \rightarrow_{\Sigma_{1}} 3$ and thus the first candidate is the Condorcet winner.

We finish this section with another measure which aims to capture the structure of relations between the different candidates. For two permutations $\sigma, \pi \in \mathbb{S}_{m}, d_{\tau}(\sigma, \pi)$ is the Kendall's $\tau$ distance between $\sigma$ and $\pi$, which is the minimal number of adjacent transpositions to change $\sigma$ into $\pi$. Given a ranking profile, $\Sigma=\left(\sigma_{1}, \ldots, \sigma_{n}\right)$, the sum

$$
\Psi(\Sigma)=\sum_{k=1, \ell=k+1}^{n} d_{\tau}\left(\sigma_{k}, \sigma_{\ell}\right)
$$

is called the profile potential of $\Sigma$. Finally, for $1 \leqslant i<j \leqslant$ $m, w_{i, j}$ is the number of voters who rank the $i$-th candidate higher than the $j$-th candidate and $q_{i, j}=\frac{w_{i, j}}{n}$. The profile potential entropy is defined to be $\sum_{i=1, j=i+1}^{m} \mathcal{H}\left(q_{i, j}\right)$.

\section{BASIC PROPERTIES}

In this section we study some basic properties on the tools introduced in Section II. We start with a basic observation on the size of the Smith set and the global intransitivity property.

Lemma 1. The Smith set of a profile $\Sigma$ is $[m]$ if and only if it has global intransitivity.

Proof: Assume $\Sigma$ has global intransitivity then (by definition) it has a cycle of length $m$. Assume without loss of generality that the cycle is $1 \rightarrow_{\Sigma} 2 \rightarrow_{\Sigma} 3 \rightarrow_{\Sigma} \cdots \rightarrow_{\Sigma} m \rightarrow_{\Sigma} 1$. Let $S$ be the Smith set of $\Sigma$ and assume in contradiction that $S \neq[m]$. Let $i$ be such that $i \in S$. Since candidate $i-1$ is winning candidate $i$, we conclude that $i-1 \in S$. Similarly $i-2 \in S$ and so going on the opposite directions of the cycle 
we get that every candidate belongs to the cycle, which leads to a contradiction.

Assume now that the Smith set of $\Sigma$ is $[m]$ and assume in contradiction that the size of the longest cycle is $\ell<m$,

$$
j_{1} \rightarrow_{\Sigma} j_{2} \rightarrow_{\Sigma} j_{3} \rightarrow_{\Sigma} \cdots \rightarrow_{\Sigma} j_{\ell} \rightarrow_{\Sigma} j_{1} .
$$

Let $S=\left\{j_{1}, \ldots, j_{\ell}\right\}$ and we distinguish between two cases: Case 1: There is a candidate $i \notin S$ which is winning at least one candidate in $S$ and there is at least one candidate in $S$ which is winning $i$. In this case we can find two consecutive candidates in the cycle, say $j_{k}$ and $j_{k+1}$ such that $i$ is winning $j_{k+1}$ and $j_{k}$ is winning $i$. Then we can extend the cycle of length $\ell$, which leads to a contradiction.

Case 2: Every candidate $i \notin S$ is either winning all the candidates in $S$ or is losing to all the candidates in $S$. Let $W$ be the set of all candidates of the first kind, and $L$ the set of the candidates of the second kind. Note that since the Smith set is $[m]$ neither of these sets is empty. Furthermore, there is a candidate $\ell \in L$ who is winning a candidate $w \in W$; otherwise $W$ is the Smith set, in contradiction. Now we can extend the cycle of length $\ell$ with the $w$-th and $\ell$-th candidates.

Next, we turn to evaluate the expected values of the measurements we introduced before. Since for every $1 \leqslant i \leqslant$ $m, H R_{\Sigma}(i) \leqslant \log m$, we immediately have that $H(\Sigma) \leqslant$ $m \log m$. The next lemma states the expected value of the profile entropy under the assumption that every ranking is chosen uniformly at random from $\mathbb{S}_{m}$.

Lemma 2. Assume every voter chooses its ranking uniformly at random from $\mathbb{S}_{m}$ of all permutations. Then, the expected value of the profile entropy satisfies

$$
m \log m-m \log (1+(m-1) / n) \leqslant E[H(\Sigma)] \leqslant m \log m .
$$

From Lemma 2, we deduce that for $n$ large enough $E[H(\Sigma)] \approx m \log m$. In order to perform a similar calculation for the profile potential we state a useful property.

Proposition 3 For any profile $\Sigma=\left(\sigma_{1}, \ldots, \sigma_{n}\right)$, we have

$$
\Psi(\Sigma)=\sum_{i=1, j=i+1}^{m} w_{i, j}\left(n-w_{i, j}\right) .
$$

From Proposition 3, we conclude that the maximum value of the profile potential value is $\left(\begin{array}{c}m \\ 2\end{array}\right) \cdot\left(\frac{n}{2}\right)^{2}$, in case $n$ is even and $\left(\begin{array}{c}m \\ 2\end{array}\right) \cdot\left(\frac{n-1}{2} \cdot \frac{n+1}{2}\right)$, in case $n$ is odd. Using this proposition we can calculate the expected value of the profile potential and show that $E[\Psi(\Sigma)]=\frac{1}{2}\left(\begin{array}{c}m \\ 2\end{array}\right)\left(\begin{array}{c}n \\ 2\end{array}\right)$.

\section{Global Intransitivity}

Our goal in this section is to better understand, using the measures introduced in Section II, the implications of global intransitivity. In general, we expect to see that global intransitivity implies a larger measure. However, as we shall see, a large profile measure does not characterize global intransitivity. For example, it is possible to have a profile with measure approaching the maximum value and yet without global intransitivity. To see this phenomena for the measure of profile entropy, assume there are $n=n^{\prime} m !+1$ voters. Every permutation $\sigma \in \mathbb{S}_{m}$ has $n^{\prime}$ voters which assign the ranking $\sigma$. Finally the last voter assigns the ranking $12 \cdots m$. It is straightforward to verify that this profile has a Condorcet winner and in particular does not have global intransitivity. Furthermore, if $m$ is fixed and $n$ is large enough then the entropy of every ranking and candidate approaches $\log m$, and thus the profile entropy approaches the maximum value $m \log m$. In the rest of the section we will be interested to analyze lower bounds on the profile entropy and profile potential values for profiles that have global intransitivity.

\section{A. The profile entropy measure}

Our first result is in a sense a negative one that shows that the profile entropy does not capture global intransitivity well.

Theorem 4. If a profile $\Sigma$ of $m$ candidates has global intransitivity then $H(\Sigma) \geqslant 2$. Moreover, there exists a profile $\Sigma$ of $m$ candidates with global intransitivity and $H(\Sigma)$ which asymptotically (in $n$ ) approaches 2.

Proof: Let us consider the first ranking. Since $\Sigma$ has global intransitivity, it cannot have a Condorcet winner and thus $p_{1, j} \leqslant 1 / 2$ for $1 \leqslant j \leqslant m$. Therefore,

$H R_{\Sigma}(1)=\mathcal{H}\left(p_{1,1}, \ldots, p_{1, m}\right)=\sum_{j=1}^{m} p_{1, j} \log \frac{1}{p_{1, j}} \geqslant \sum_{j=1}^{m} p_{1, m}=1$.

Similarly, there is no Condorcet loser and so $H R_{\Sigma}(m) \geqslant 1$. Finally, we get $H(\Sigma) \geqslant H R_{\Sigma}(1)+H R_{\Sigma}(m) \geqslant 2$.

To show that this bound is tight we present the following profile $\Sigma_{2}$ with $n=2 n^{\prime}+1$ voters where $n^{\prime}$ of them have the ranking $(1,2,3, \cdots, m-1, m), n^{\prime}$ more have the ranking $(m, 2,3, \cdots, m-1,1)$, and the last voter has the ranking $(m-$ $1, m, 1,2, \cdots, m-2)$. It can be verified that the Smith set of $\Sigma_{2}$ is $[m]$ and in particular there is no Condorcet winner. The probability ranking matrix is

$$
P_{\Sigma_{2}}=\left(\begin{array}{ccccccc}
\frac{n^{\prime}}{n} & 0 & 0 & \cdots & 0 & \frac{1}{n} & \frac{n^{\prime}}{n} \\
0 & \frac{n-1}{n} & 0 & \cdots & 0 & 0 & \frac{1}{n} \\
\frac{1}{n} & 0 & \frac{n-1}{n} & \cdots & 0 & 0 & 0 \\
\vdots & \vdots & \vdots & \vdots & \vdots & \vdots & \vdots \\
0 & 0 & 0 & \cdots & 0 & \frac{n-1}{n} & 0 \\
\frac{n^{\prime}}{n} & 0 & 0 & \cdots & \frac{1}{n} & 0 & \frac{n^{\prime}}{n}
\end{array}\right) .
$$

Finally, for $n$ large enough $H\left(\Sigma_{2}\right)=H R_{\Sigma_{2}}(1)+H R_{\Sigma_{2}}(m)+$ $o(1)$ which approaches 2 as $n$ increases.

The last result is somehow disappointing. We can find a a cycle of $m$ candidates where the entropy profile approaches two, that is, independently with the number of candidates. Our next step is to draw more connections with the number of candidates and in particular with the structure of the cycles between the candidates. A first observation on the structure of the cycles is derived as follows. We define a 3-cycle to be a cycle between three candidates.

Lemma 5. Assume a profile $\Sigma$ has a cycle of length $\ell$, then it has a 3-cycle. In fact, every candidate in the cycle belongs to at least one 3-cycle.

Proof: Let us denote the candidates by $1,2, \ldots, \ell$ and without loss of generality assume there is a cycle of the form $1 \rightarrow_{\Sigma} 2 \rightarrow_{\Sigma} 3 \rightarrow_{\Sigma} \cdots \rightarrow_{\Sigma} \ell \rightarrow_{\Sigma} 1$. We will show that the 
first candidate belongs to some 3-cycle. If candidate 3 is winning candidate 1 , then there is a 3 -cycle between candidates 1,2 , and 3. Otherwise, we found a smaller cycle of length $m-1,1 \rightarrow_{\Sigma} 3 \rightarrow_{\Sigma} \cdots \rightarrow_{\Sigma} m \rightarrow_{\Sigma} 1$. We can continue with the same argument until we reach the case of a four cycle $1 \rightarrow_{\Sigma} m-2 \rightarrow_{\Sigma} m-1 \rightarrow_{\Sigma} m \rightarrow_{\Sigma} 1$, which yields to one of the following 3-cycles: $1 \rightarrow_{\Sigma} m-2 \rightarrow_{\Sigma} m-1 \rightarrow_{\Sigma} 1$ or $1 \rightarrow_{\Sigma} m-1 \rightarrow_{\Sigma} m \rightarrow_{\Sigma} 1$.

In the profile $\Sigma_{2}$ given in the proof of Theorem 4 , we can see that indeed every candidate belongs to at least one 3-cycle. However all 3-cycles are of the form $m-1 \rightarrow_{\Sigma} m \rightarrow_{\Sigma} i \rightarrow_{\Sigma}$ $m-1$ or $m-1 \rightarrow_{\Sigma} 1 \rightarrow_{\Sigma} i \rightarrow_{\Sigma} m-1$, for $2 \leqslant i \leqslant m-2$. In particular, it is not possible to find two candidate-disjoint 3 -cycles. We will show how the number of candidate-disjoint 3-cycles affects the profile entropy.

Lemma 6. Assume a profile $\Sigma$ has $\ell \geqslant 2$ disjoint 3-cycles between the $m$ candidates, then $H(\Sigma) \geqslant \ell$. Moreover, there is a profile $\Sigma$ with $\ell$ disjoint 3-cycles between the $m$ candidates in which $H(\Sigma)$ approaches $\ell$ (with large $n$ ).

Proof: Assume candidates $i, j, k$ form a 3-cycle. Let $f$ be the following function $f:\{1, \ldots, m\}^{3} \rightarrow \mathbb{S}_{3}$, which converts the three rankings of the candidates $i, j, k$ to a relative ranking between them as three candidates. Let $X_{i}, X_{j}, X_{k}$ be a random variable of the ranking of candidate $i, j, k$, respectively, and note that $H C_{\Sigma}(i)=\mathcal{H}\left(X_{i}\right), H C_{\Sigma}(j)=\mathcal{H}\left(X_{j}\right), H C_{\Sigma}(k)=$ $\mathcal{H}\left(X_{k}\right)$. Since $f$ is a one to one function, we have that

$$
\mathcal{H}\left(X_{i}, X_{j}, X_{k}\right) \geqslant \mathcal{H}\left(f\left(X_{i}, X_{j}, X_{k}\right)\right) .
$$

The variable $f\left(X_{i}, X_{j}, X_{k}\right)$ corresponds to a permutation in $\mathbb{S}_{3}$. Since there is a cycle between the three candidates, there is no permutation of $f\left(X_{i}, X_{j}, X_{k}\right)$ which appears more than half of the times. Therefore, similarly to the proof of Theorem 4 we can show that $\mathcal{H}\left(f\left(X_{i}, X_{j}, X_{k}\right)\right) \geqslant 1$. Finally,

$$
\begin{aligned}
& H C_{\Sigma}(i)+H C_{\Sigma}(j)+H C_{\Sigma}(k)=\mathcal{H}\left(X_{i}\right)+\mathcal{H}\left(X_{j}\right)+\mathcal{H}\left(X_{k}\right) \\
& \geqslant \mathcal{H}\left(X_{i}, X_{j}, X_{k}\right) \geqslant \mathcal{H}\left(f\left(X_{i}, X_{j}, X_{k}\right)\right) \geqslant 1 .
\end{aligned}
$$

To conclude, we apply this argument to each one of the $\ell$ 3cycles and get that the profile entropy is at least $\ell$.

To prove that the entropy lower bound is tight, let $\Sigma_{3}$ be a profile with six candidates and $n=2 n^{\prime}+1$ voters. The first $n^{\prime}$ voters assign the ranking $(3,1,4,5,2,6)$, the next $n^{\prime}$ voters assign the ranking $(6,1,4,5,2,3)$, and the last voter assigns the ranking $(2,3,1,5,6,4)$. It is possible to verify that there is a 3-cycle between candidates 1, 2, and 3 and another 3-cycle between candidates 4,5 , and 6 . The entropy sum of the six candidates satisfies $H(\Sigma) \geqslant 1+1=2$, and $H(\Sigma)$ approaches 2 as $n$ gets large.

In general, this example can be extended for $6 \mathrm{~m}$ candidates such that the number of disjoint 3-cycles is $2 m$, which is maximized, and the profile entropy approaches $2 \mathrm{~m}$.

\section{B. The profile potential measure}

We now turn to study the profile potential.

Theorem 7. Assume a profile $\Sigma$ has global intransitivity, then the profile potential satisfies $\Psi(\Sigma) \geqslant \frac{m-1}{4} n^{2}$. Moreover, there exists a profile $\Sigma$ with global intransitivity for which $\Psi(\Sigma)<$ $\frac{m-1}{4} n^{2}+n(m-2)$.

Proof: Let us first consider a cycle between three candidates. Assume without loss of generality that candidate 1 is winning candidate 2 , candidate 2 is winning candidate 3 , and candidate 3 is winning candidate 1 . Assume also that $w_{1,2}=$ $n / 2+a$ and $w_{2,3}=n / 2+b$, where $a, b \geqslant 0$. Therefore, we have that $a+b \leqslant w_{1,3} \leqslant n / 2$ and thus

$$
\begin{aligned}
& w_{1,2}\left(n-w_{1,2}\right)+w_{2,3}\left(n-w_{2,3}\right)+w_{1,3}\left(n-w_{1,3}\right) \\
& =(n / 2)^{2}-a^{2}+(n / 2)^{2}-b^{2}+w_{1,3}\left(n-w_{1,3}\right) \\
& \geqslant(n / 2)^{2}-a^{2}+(n / 2)^{2}-b^{2}+(a+b)(n-(a+b)) \\
& \geqslant n^{2} / 2+n(a+b)-2(a+b)^{2} \\
& =n^{2} / 2+2(a+b)(n / 2-(a+b)) \geqslant n^{2} / 2,
\end{aligned}
$$

where the last inequality follows from $a+b \leqslant n / 2$.

The proof for arbitrary $m$ holds by induction. The base for three candidates was proved above. Assume the claim is true for $m-1$ candidates and we will prove the correctness for $m$ candidates. Let the cycle between the $m$ candidates be $1 \rightarrow_{\Sigma}$ $2 \rightarrow_{\Sigma} \cdots \rightarrow_{\Sigma} m \rightarrow_{\Sigma} 1$. We distinguish between two cases: 1. there is a cycle of between $m-1$ candidates, and 2 . we cannot find a cycle between $m-1$ candidates.

Case 1: Assume without loss of generality that these are the first $m-1$ candidates. According the induction assumption and Proposition $3 \sum_{i=1, j=i+1}^{m-1} w_{i, j}\left(n-w_{i, j}\right) \geqslant \frac{m-2}{4} n^{2}$. According to Lemma 5 , the $m$-th candidate belongs to a 3-cycle and assume it includes the $k$-th and $\ell$-th candidates. As proved in the beginning of this proof, $w_{k, \ell}\left(n-w_{k, \ell}\right)+w_{\ell, m}\left(n-w_{\ell, m}\right)+$ $w_{k, m}\left(n-w_{k, m}\right) \geqslant n^{2} / 2$, and since $w_{k, \ell}\left(n-w_{k, \ell}\right) \leqslant n^{2} / 4$, we have $w_{\ell, m}\left(n-w_{\ell, m}\right)+w_{k, m}\left(n-w_{k, m}\right) \geqslant n^{2} / 4$. Together we conclude that $\Psi(\Sigma)=\sum_{i=1, j=i+1}^{m} w_{i, j}\left(n-w_{i, j}\right)$ is at least

$$
\begin{aligned}
& \sum_{i=1, j=i+1}^{m-1} w_{i, j}\left(n-w_{i, j}\right)+w_{\ell, m}\left(n-w_{\ell, m}\right)+w_{k, m}\left(n-w_{k, m}\right) \\
\geqslant & \frac{m-2}{4} n^{2}+\frac{n^{2}}{4}=\frac{m-1}{4} n^{2} .
\end{aligned}
$$

Case 2: If there is no cycle between any $m-1$ candidates, then for $1 \leqslant i \leqslant m, i+2 \rightarrow_{\Sigma} i$ (in case an index is greater than $m$ we take its residue $\bmod (m)$ as a number between 1 and $m$ ). Therefore, we get $m$ different cycles of length 3 , $i \rightarrow_{\Sigma} i+1 \rightarrow_{\Sigma} i+2 \rightarrow_{\Sigma} i$, and thus $W_{i}=w_{i, i+1}(n-$ $\left.w_{i, i+1}\right)+w_{i+1, i+2}\left(n-w_{i+1, i+2}\right)+w_{i, i+2}\left(n-w_{i, i+2}\right) \geqslant n^{2} / 2$. Finally, we conclude with the following

$$
\Psi(\Sigma) \geqslant \frac{1}{2} \sum_{i=1}^{m} W_{i} \geqslant \frac{1}{2} \cdot m \cdot \frac{n^{2}}{2}>\frac{m-1}{4} n^{2}
$$

To show that the lower bound is tight we present the following example. Assume a profile $\Sigma$ with $n=2 n^{\prime}+1$ voters and $m$ candidates. The first $n^{\prime}$ voters have the ranking $\sigma_{1}=(1,2, \ldots, m)$, the next $n^{\prime}$ voters have the ranking $\sigma_{2}=$ $(m, 1,2, \ldots, m-1)$, and the last voter has the ranking $\sigma_{3}=$ $(m-1, m, 1, \ldots, m-2)$. There is a cycle between these $m$ candidates and thus the profile has global intransitivity. It can be verified that the profile potential satisfies $\Psi(\Sigma)<\frac{m-1}{4} n^{2}+$ $n(m-2)$.

It can be shown that the example profile presented in the proof of Theorem 7 has profile potential entropy approaching 
$m-1$. Similarly to the proof of Theorem 7 it is possible to show that if a profile has global intransitivity then its profile potential entropy is at least $m-1$. We omit the details due to the lack of space.

\section{RANKINGS IN A BALL}

In this section we study a special case where the voters can choose their rankings only within a special set, which is ball of a prescribed radius according to the Kendall's $\tau$ distance. The goal is to study the largest radius of a ball such that there is no global intransitivity for any profile. The motivation to restrict the profile rankings to belong solely to a ball comes from the distance-based models for ranking data [4]. Under such models, the probability of each ranking is a function of some center permutation and the distance from this center.

The problem we study here is a variation of the Condorcet domain problem. A Condorcet domain is a set $S \subseteq \mathbb{S}_{m}$ of permutations such that every profile $\Sigma \in S^{n}$ has a Condorcet winner. The problem of finding the largest size of a Condorcet domain is fascinating by itself and in fact is still an open problem for arbitrary $m$; see e.g. [2], [7]. However, this problem is out of the scope of this paper.

We relax the requirement of having a Condorcet winner and ask that there will not be global intransitivity. A ball with radius $r$ and center $\sigma \in \mathbb{S}_{m}$ is the set $B_{r}(\sigma)=\left\{\pi \mid d_{\tau}(\sigma, \pi) \leqslant\right.$ $r$. We define $R(m)$ to be the largest radius such that for any ball $B_{R(m)}(\sigma)$, every profile $\Sigma \in\left(B_{R(m)}(\sigma)\right)^{n}$ does not have global intransitivity.

Theorem 7 already gives us an example where the permutations of the profile $\Sigma$ can be located inside a ball of radius $m-1$, centered in $\sigma_{2}$ and yet there is a cycle between all $m$ candidates. Therefore, $R(m)<m-1$. On the other hand, if $R(m)=\lfloor(m-1) / 4\rfloor$ then for every pair of permutations $\sigma_{i}$ and $\sigma_{j}$ in the profile $\Sigma, d_{\tau}\left(\sigma_{i}, \sigma_{j}\right) \leqslant(m-1) / 2$ and thus the profile potential in this case satisfies $\Psi(\Sigma) \leqslant$ $\left(\begin{array}{l}n \\ 2\end{array}\right)(m-1) / 2<\frac{m-1}{4} n^{2}$. Therefore, according to Theorem 7 the there is no global intransitivity. Hence, we conclude so far that $\left\lfloor\frac{m-1}{4}\right\rfloor \leqslant R(m) \leqslant m-2$. We will now show how to close on this gap and obtain

Theorem 8. For all $m \geqslant 3, R(m)=\lceil m / 2\rceil$.

We first prove a lower bound and then an upper bound.

Lemma 9. For all $m \geqslant 3, R(m) \geqslant\lceil m / 2\rceil$.

Proof: Due to the lack of space we show the proof only for odd values of $m$. The proof for even values will follow similar guidelines. Without loss of generality assume that the center of the ball is the identity permutation $\pi=123 \cdots m$. Let $\Sigma=\left(\sigma_{1}, \ldots, \sigma_{n}\right)$ be a profile in $B_{\frac{m+1}{2}}(\pi)$ and assume in contradiction that the Smith set of $\Sigma$ is $[m]$. Let us assume for now that the cycle between the $m$ candidates is the following $m \rightarrow_{\Sigma} m-1 \rightarrow_{\Sigma} m-2 \rightarrow_{\Sigma} \cdots \rightarrow_{\Sigma} 2 \rightarrow_{\Sigma} 1 \rightarrow_{\Sigma} m$. For $1 \leqslant i \leqslant m-1$, let $w_{i+1, i}=\left|\left\{\sigma \mid i+1>_{\sigma} i\right\}\right|$, and $w_{i, i+1}=\left|\left\{\sigma \mid i>_{\sigma} i+1\right\}\right|$. Note that $w_{i+1, i}+w_{i, i+1}=n$. Then, this cycle results with the following $m-1$ inequalities: $w_{i+1, i}>w_{i, i+1}$, for $1 \leqslant i \leqslant m-1$. In particular, we get that $\sum_{i=1}^{m-1} w_{i+1, i}>\sum_{i=1}^{m-1} w_{i, i+1}=n(m-1)-\sum_{i=1}^{m-1} w_{i+1, i}$ and therefore,

$$
\sum_{i=1}^{m-1} w_{i+1, i}>n(m-1) / 2 .
$$

For any permutation $\sigma$, let $w_{\sigma}=\mid\left\{1 \leqslant i \leqslant m-1 \mid i+1>_{\sigma}\right.$ $i\} \mid$. Then, $\sum_{i=1}^{m-1} w_{i+1, i}=\sum_{\ell=1}^{n} w_{\sigma_{\ell}}$. It can be shown that if $d_{\tau}\left(\pi, \sigma_{\ell}\right) \leqslant \frac{m+1}{2}$ then $w_{\sigma_{\ell}} \leqslant \frac{\ell-1}{2}$. Therefore,

$$
\sum_{i=1}^{m-1} w_{i+1, i}=\sum_{\ell=1}^{n} w_{\sigma_{\ell}} \leqslant n(m-1) / 2,
$$

in contradiction to (1). The proof for other types of cycles is very similar and thus we omit the details.

Lemma 10. For all $m \geqslant 3, R(m) \leqslant\lceil m / 2\rceil$.

Proof: We will show an example of a ball with radius $\lceil m / 2\rceil+1$ and a profile $\Sigma$ with rankings only from this ball such that its Smith set is $[m]$.

We assume the center of the ball is the identity permutation $\pi=123 \cdots m$ and its radius is $\lceil m / 2\rceil+1$. Due to space limitations, we present here a proof for the case $m=9$ which exhibits the intuition of the proof for general $m$ (which follows very similar lines). In the following profile, we underline consecutive locations in the permutations that are subject to transpositions. The pattern of the subsets of indices that are subject to transpositions should be clear from the example.

$$
\begin{aligned}
& \sigma_{1}=\underline{3,2,1,5,4,7,6,9,8} \quad \sigma_{2}=1,4,3,2,6,5,8,7,9 \\
& \sigma_{3}=2,1,5,4,3,7,6,9,8 \quad \sigma_{4}=1,3,2,6,5,4,8,7,9 \\
& \sigma_{5}=2,1,4,3,7,6,5,9,8 \quad \sigma_{6}=1,3,2,5,6,8,7,6,9 \\
& \sigma_{7}=\underline{2,1}, \underline{4,3}, \underline{6,5}, \underline{9,8,7}
\end{aligned}
$$

In this example, the distance between every $\sigma_{i}$ and 123456789 is at most $6=\left\lceil\frac{9}{2}\right\rceil+1$, and there is a cycle $9 \rightarrow_{\Sigma} 8 \rightarrow_{\Sigma}$ $7, \ldots, 2 \rightarrow_{\Sigma} 1 \rightarrow_{\Sigma} 9$.

\section{ACKNOWLEDGMENTS}

This work was partially supported by NSF grant ECCS0801795 and BSF grant 2010075.

\section{REFERENCES}

[1] M.D. Condorcet, Essai sur l'application de l'analyse à la probabilité des decisions rendues à la pluralité des voix (Essay on the Application of Probability Analysis to Majority Decision), Paris, 1785.

[2] P.C. Fishburn, "Acyclic sets of linear orders: a progress report," Social Choise and Welfare, vol. 19, pp. 431-437, 2002.

[3] J.Hodge and R.E. Klima, "The mathematics of voting and elections: a hands-on approach," Mathematical World Series, vol.22. Am. Math. Soc., 2005.

[4] T.B. Murphya and D. Martinb, "Mixtures of distance-based models for ranking data,'Computational Statistics and Data Analysis, vol.41, pp. 645-655, 2003

[5] A.D. Procaccia, "Computational social choice: the first four centuries," ACM XRDS, vol. 18, no. 2, pp.31-34, November 2011.

[6] M. Regenwetter, B. Grofman, A.A.J. Marley, and I.M. Tsetlin, Behavioral Social Choice, Cambridge University Press, 2006.

[7] W.H. Riker, Liberalism against Populism, W. H. Freeman and Co., San Francisco, 1982.

[8] K. Shepsle and M. Bonchek, Analyzing Politics: Rationality, Bhavior and Institutions, Norton, New York, 1997.

[9] J.H. Smith, "Aggregation of preferences with variable electorate," Econometrica vol. 41, no. 6, pp. 1027-1041, November 1973.

[10] A.S. Tangian, "Unlikelihood of Condorcet's paradox in a large society," Soc. Choice Welfare, vol. 17, pp. 337-365, 2000. 